\title{
On the Coupling Integrals Arising in the Method of Moments Formulation of Laterally Bounded Structures
}

\author{
Pedro Crespo-Valero, Ivica Stevanović, Member, IEEE, \\ Daniel Llorens del Río, Member, IEEE, and Juan R. Mosig, Fellow, IEEE
}

\begin{abstract}
A generic integral equation and method of moments formulation is presented for laterally bounded stratified media including planar metallization. The main asset of the developed approach is its flexibility, as it encompasses generic lateral boundary conditions and explicitly applies to any linear subsectional basis functions with constant surface divergence. This includes the rooftop functions on rectangular and triangular supports currently proposed in standard method of moment meshers. This approach provides closed expressions for the coupling integrals appearing in the method of moments matrix elements. These formulas are based of Green's functions modal expansions and in the possibility, conclusively demonstrated in this paper to transform the surface integrals into contour integrals allowing an efficient and systematic implementation of the procedure. Full derivations are presented for several lateral boundary conditions, including rectangular and circular metallic cavities and periodic structures. Numerical examples including the analysis of real-life planar boxed circuits are presented. In all cases the obtained results compare favourably with other existing techniques.
\end{abstract}

Index Terms-Coupling integrals, Green's function, integral equation, lateral boundary conditions, method of moments (MoM), periodic structures, planar circuits, stratified media, waveguides.

\section{INTRODUCTION}

$\mathbf{P}$ RACTICAL RF, microwave, and high-speed electronic circuits are typically realized with planar transmission lines and components printed over dielectric layers. The electromagnetic (EM) modeling of these structures has been subject of study for a long time, yielding a variety of numerical methods. Despite the power of generic numerical techniques like finite elements [1], finite differences [2] or the transmission-line modeling [3] methods, the techniques based on integral equations in conjunction with the method of moments (MoM) have shown

Manuscript received July 06, 2008; revised September 08, 2008. First published November 18, 2008; current version published December 05, 2008.

P. Crespo-Valero is with the Research and Development Software Group, Schmid \& Partner Engineering AG (SPEAG), CH-8004 Zurich, Switzerland (e-mail: crespo@speag.com).

I. Stevanović is with Freescale Semiconductor, CH-1211 Geneva, Switzerland (e-mail: ivica.stevanovic@ freescale.com).

D. Llorens del Río is with JAST Antenna Systems, CH-1015 Lausanne, Switzerland (e-mail: daniel.llorensdelrio@jast.ch)

J. R. Mosig is with the Laboratory of Electromagnetics and Acoustics (LEMA), Ecole Polytechnique Fédérale de Lausanne (EPFL), CH-1015 Lausanne, Switzerland (e-mail: juan.mosig@epfl.ch).

Color versions of one or more of the figures in this paper are available online at http://ieeexplore.ieee.org.

Digital Object Identifier 10.1109/TMTT.2008.2007327 to be the most efficient for this kind of structures [4]-[6]. The strength of integral equation techniques lies in the formulation of an ad hoc Green's function that provides an EM description of the surrounding environment, reducing the number of unknowns in the numerical problem and improving the accuracy of the solution. This advantage, however, is at the same time its biggest drawback compared to the aforementioned methods, since every different environment will require a completely different Green's function formulation.

In this study, we mitigate this constraint and present a generic formulation for planar circuits within laterally bounded media, with a seamless transition between different lateral boundary conditions. The concept of laterally bounded media accounts for any structure having a boundary condition with uniaxial symmetry wrapping the planar circuit. This allows an efficient analysis of a wide range of useful structures such as planar circuits embedded in shielded multilayered media, infinitely thin discontinuities in waveguides or even 2-D printed periodic structures.

These examples were solved using different integral equation-MoM approaches. A classic strategy is to derive specialized Green's function for each particular boundary condition while the currents of the planar circuits are approximated as an expansion of basis functions. Typically, the Green's function of bounded problems with canonical shapes can be computed using quasi-analytical expressions (e.g., expansion of modes in a rectangular/circular waveguide), whereas more intricate shapes can be treated using numerical approaches [7]. There are also different choices of basis functions depending on the shape of the planar circuits. For arbitrary shapes subsectional basis functions [8] are preferred whereas entire domain basis functions might be a more suitable choice for circuits having a canonical geometrical form [9]. An intermediate solution to deal with 'nearly-canonical' shapes is also provided in [10], [11] using the boundary integral resonant-mode expansion method. Finally, the combination of these elements in the MoM led to different integration schemes to be solved for each scenario: each problem required a different method.

The formulation that we present here is able to provide a systematic approach in the most general case, i.e., assuming arbitrary shape for both the planar circuits and the boundary conditions. This technique is based on the modal representation of the fields in bounded media and uses general properties satisfied by a wide range of practical basis functions. This combination results in a generic MoM scheme with coupling integrals, i.e., 


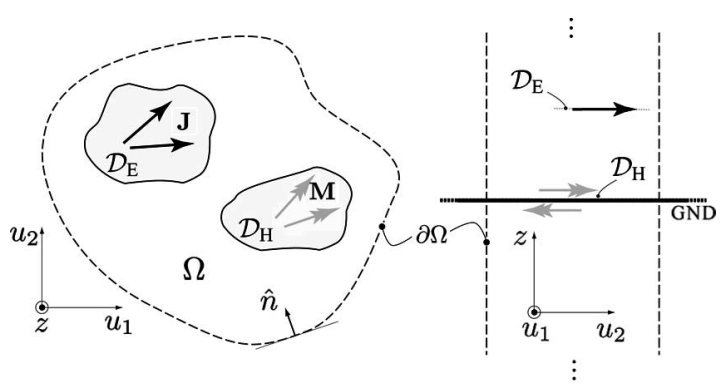

(a)

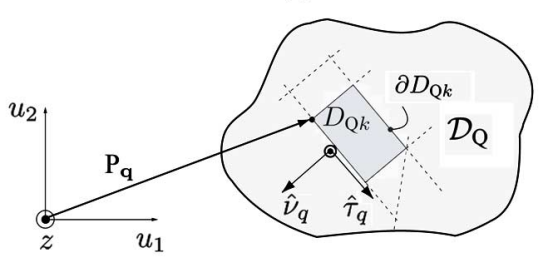

(b)

Fig. 1. (a) Cross section (left) and longitudinal view (right) of planar electric (J) and magnetic (M) sources embedded in a medium with boundary condition imposed on an arbitrary cylindrical surface $\partial \Omega$. (b) Segmentation of plane surface with $\mathrm{Q}=\{\mathrm{E}, \mathrm{H}\}$-type sources into $D$ domains.

integrals between a mode and a basis function, that can be reduced to contour integrals. The evaluation of reaction terms in the MoM matrix is therefore greatly simplified, resulting also in analytical solutions to the coupling integrals when generic boundary conditions are considered over canonical shapes.

This paper is organized as follows. It starts with an overview of the integral equation and MoM formulation in order to provide a working frame and to introduce the notation. This section concludes with a formal definition of the coupling integrals that are the cornerstone of this approach. The next section describes how the coupling integral expressions can be reduced from surface to contour integrals. This results in a very general and powerful algorithmic framework. For the sake of concreteness, two specific specializations are considered. Firstly, our formulation is shown to be applicable without modification to two of the most commonly used subsectional basis functions. Secondly, three different boundary conditions are considered, namely rectangular and circular perfect electric conductor (i.e., waveguides) and periodic boundary conditions. Finally, the validity of our approach is convincingly demonstrated by applying it to several structures.

\section{INTEGRAL EQUATION-MoM FORMULATION}

The transverse and longitudinal cross sections of a generic planar structure in a bounded layered medium are depicted in the left and right hand side of Fig. 1(a), respectively. The planar structures are represented as two arbitrary surfaces, $\mathcal{D}_{\mathrm{E}}$ and $\mathcal{D}_{\mathrm{H}}$, enclosed by a generic lateral boundary condition over $\partial \Omega$ that extends infinitely along the $z$ axis. The surface $\mathcal{D}_{\mathrm{E}}$ stands for any planar metallic (ideally perfect electric conductor) obstacles isolated at a certain height of the cylindrical structure, whereas $\mathcal{D}_{\mathrm{H}}$ corresponds to a generic slot or aperture drilled on a ground plane transverse to the boundaries, as depicted in the longitudinal cross section of Fig. 1(a).
Using the equivalence principle [5], [12], EM scattering on these surfaces can be modeled by replacing them with equivalent transverse electric $\mathbf{J} \in \mathcal{D}_{\mathrm{E}}$ and magnetic $\mathbf{M} \in \mathcal{D}_{\mathrm{H}}$ induced surface currents that radiate in the medium. Slots are replaced by continuous ground planes and magnetic currents, identical but of opposite sign, must be introduced on both sides of the ground plane at the points previously occupied by the slot [4]. This is represented in Fig. 1(a) with arrows over the planar surfaces indicating the current flow.

The total EM field can then be recovered as the superposition of an excitation field and the scattered field, radiated by $\mathbf{J}$ and $\mathbf{M}$ in the equivalent problem and can be written as

$$
\begin{aligned}
\mathbf{E}^{\mathrm{T}} & =\mathbf{E}^{\mathrm{e}}+\mathbf{E}^{\mathrm{s}}(\mathbf{J}, \mathbf{M}) \\
\mathbf{H}^{\mathrm{T}} & =\mathbf{H}^{\mathrm{e}}+\mathbf{H}^{\mathrm{s}}(\mathbf{J}, \mathbf{M})
\end{aligned}
$$

where the superscripts T, e, and s stand for total, excitation, and scattered, respectively. From now on, we will consider exclusively tangential fields. Hence, $\{\mathbf{E}, \mathbf{H}\}$ will denote the tangential components of the electric and magnetic fields. The next step towards the establishment of an integral equation formulation is to consider the boundary conditions to be satisfied by the tangential fields. A careful distinction must be made here between metallic surfaces $\mathcal{D}_{\mathrm{E}}$ and apertures on metallic ground planes $\mathcal{D}_{\mathrm{H}}$. In the first case, the relevant boundary condition is a vanishing total tangential electric field

$$
\mathbf{E}^{\mathrm{e}}+\mathbf{E}^{\mathrm{S}}(\mathbf{J}, \mathbf{M})=0 \text { on } \mathcal{D}_{\mathrm{E}} .
$$

On the other hand, continuity of both electric and magnetic total tangential fields must be imposed for the slots. Selecting equivalent magnetic currents equal but of opposite sign at both sides of the slot already guarantees the continuity of the total tangential electric field. The continuity of the magnetic field must be explicitly imposed in a boundary condition. Using indices I and II to denote the two sub-regions at both sides of the slot, this boundary condition can be written as

$$
\mathbf{H}_{\mathrm{I}}^{\mathrm{e}}+\mathbf{H}_{\mathrm{I}}^{\mathrm{s}}\left(\mathbf{J}_{\mathrm{I}},-\mathbf{M}\right)=\mathbf{H}_{\mathrm{II}}^{\mathrm{e}}+\mathbf{H}_{\mathrm{II}}^{\mathrm{s}}\left(\mathbf{J}_{\mathrm{II}}, \mathbf{M}\right) \text { on } \mathcal{D}_{\mathrm{H}} \text {. }
$$

For the sake of compactness and internal coherence, we introduce the symbol $\Delta f$ to denote the difference $f_{\mathrm{I}}-f_{\mathrm{II}}$ and we write the boundary condition (3) as

$$
\Delta \mathbf{H}^{\mathrm{e}}+\Delta \mathbf{H}_{\mathrm{I}}^{\mathrm{S}}(\mathbf{J}, \mathbf{M})=0 \text { on } \mathcal{D}_{\mathrm{H}}
$$

Thus, the boundary conditions (2) and (4) now have the same formal mathematical structure. By using linearity and the Green's function concept, the scattered field can now be obtained as

$$
\left[\begin{array}{c}
\mathbf{E}^{\mathrm{s}} \\
\Delta \mathbf{H}^{\mathrm{s}}
\end{array}\right]=\left[\begin{array}{cc}
\overleftrightarrow{\mathbf{G}}_{\mathrm{EE}} & \stackrel{\leftrightarrow}{\mathbf{G}}_{\mathrm{EH}} \\
\overleftrightarrow{\mathbf{G}}_{\mathrm{HE}} & \Delta \stackrel{\mathbf{G}}{\mathrm{HH}}
\end{array}\right] *\left[\begin{array}{c}
\mathbf{J} \\
\mathbf{M}
\end{array}\right]
$$

Again, special care must be taken when applying these generic expressions to particular situations. For instance, if we concentrate on a slot problem, the symbol $\Delta \overleftrightarrow{\mathbf{G}}_{\mathrm{HH}}$ in (5) is actually the sum and not the difference of the two Green's functions corresponding to the subproblems in both sides of the slot. This is due to the sign reversal of the equivalent magnetic currents in 
both sides of the slot. In (5), $\overleftrightarrow{\mathbf{G}}_{\mathrm{PQ}}\left(\mathbf{r} \mid \mathbf{r}^{\prime}\right)$ is the dyadic Green's function relating P-type fields at $\mathbf{r}$ and Q-type sources at $\mathbf{r}^{\prime}$ (with $\mathrm{P}, \mathrm{Q}=\{\mathrm{E}, \mathrm{H}\})$; and the operator $*$ denotes the convolution integral over the pertinent surface $\mathcal{D}_{\mathrm{Q}}$. Substituting (5) into (2) and (4) leads to the sought-after coupled system of integral equations. In the next step, these equations are further transformed into a linear system using the MoM [13]. The Galerkin procedure is applied expanding the equivalent currents as

$$
\mathbf{J}=\sum_{k} i_{k} \mathbf{b}_{k} \quad \mathbf{M}=\sum_{l} v_{l} \mathbf{b}_{l}
$$

where $i_{k}, v_{l}$ are unknown complex coefficients and $\mathbf{b}_{k} \equiv$ $\mathbf{b}\left(u_{1}^{\prime}, u_{2}^{\prime}\right)$ are basis functions defined on an arbitrary transverse domain $D_{\mathrm{Q} k} \subseteq \mathcal{D}_{\mathrm{Q}}$. It is necessary to employ expansion functions that ensure a finite divergence across domain boundaries, or equivalently basis functions that maintain normal continuity between subdomains [8]. We will consider here basis functions being locally curl-free and with locally constant charge density, i.e., any $\mathbf{b}_{k}$ satisfying

$$
\begin{aligned}
\nabla_{\mathrm{t}} \times \mathbf{b} & =0 \\
\nabla_{\mathrm{t}} \cdot \mathbf{b} & =d
\end{aligned}
$$

with $d$ being a real nonzero constant and $\nabla_{\mathrm{t}}=\nabla-\hat{z} \partial / \partial z$. These properties are satisfied by divergence-conforming basis functions [8].

The resulting linear system, discrete counterpart of our set of integral equations, is given by

$$
\left[\begin{array}{l|l}
\mathbf{R}_{\mathrm{EE}} & \mathbf{R}_{\mathrm{EH}} \\
\hline \mathbf{R}_{\mathrm{HE}} & \mathbf{R}_{\mathrm{HH}}
\end{array}\right] \cdot\left[\begin{array}{c}
\mathbf{i} \\
\mathbf{v}
\end{array}\right]=\left[\begin{array}{c}
\mathbf{u} \\
\mathbf{j}
\end{array}\right]
$$

where $\mathbf{i}, \mathbf{v}$ and $\mathbf{u}, \mathbf{j}$ are column vectors. $\mathbf{i}, \mathbf{v}$ contain the induced current coefficients defined in (6) and $\mathbf{u}, \mathbf{j}$ include the results of the projection of the excitation fields onto each basis function

$$
u_{k}=\int_{D_{\mathrm{E} k}} \mathbf{b}_{k} \cdot \mathbf{E}^{\mathrm{e}} \mathrm{d} S \quad j_{l}=\int_{D_{\mathrm{H} l}} \mathbf{b}_{l} \cdot \mathbf{H}^{\mathrm{e}} \mathrm{d} S .
$$

Finally, the four submatrices $\mathbf{R}_{\mathrm{PQ}}$ comprise the reactions between electric and magnetic surface currents [14]. Expressions can be systematically obtained for them taking into account the fact that in a laterally bounded medium, the Green's function can always be expressed as a series expansion of "guided" modes [15] as

$$
\overleftrightarrow{\mathbf{G}}_{\mathrm{PQ}}\left(\mathbf{r} \mid \mathbf{r}^{\prime}\right)=\sum_{i} \tilde{G}_{\mathrm{PQ} i}\left(z, z^{\prime}\right) \mathbf{p}_{i}\left(u_{1}, u_{2}\right) \overline{\mathbf{q}}_{i}\left(u_{1}^{\prime}, u_{2}^{\prime}\right)
$$

where $\bar{q}_{i}$ is the complex conjugate of $q_{i}$. The series (10) comprises the transverse field components $\mathbf{p}_{i}, \mathbf{q}_{i}=\left\{\mathbf{e}_{i}, \mathbf{h}_{i}\right\}$ and the spectral amplitude $\tilde{G}_{\mathrm{PQ} i}$ for the $i$ th mode. The former are eigensolutions of the transverse boundary problem (e.g., waveguide [16] or periodic structure [17]), whereas the latter is calculated by solving a transmission line model accounting for the boundary condition imposed along $z$-axis, which can be simply vacuum or a multilayered dielectric medium [18]. The same considerations apply to any linear combination of Green's functions appearing in a specific problem. Now, the reaction $R_{\mathrm{PQ}}$
TABLE I

Transverse Components in Terms of SCALAR Potentials

\begin{tabular}{|c|cc|}
\hline & $\mathbf{e}$ & $\mathbf{h}$ \\
\hline $\mathrm{TE}_{m n}$ & $\hat{z} \times \nabla_{\mathrm{t}} \chi_{m n} / \kappa_{m n}$ & $-\nabla_{\mathrm{t}} \chi_{m n} / \kappa_{m n}$ \\
\hline $\mathrm{TM}_{m n}$ & $-\nabla_{\mathrm{t}} \chi_{m n} / \kappa_{m n}$ & $-\hat{z} \times \nabla_{\mathrm{t}} \chi_{m n} / \kappa_{m n}$ \\
\hline $\mathrm{TEM}_{m}$ & $-\nabla_{\mathrm{t}} \chi_{m}^{0}$ & $-\hat{z} \times \nabla_{\mathrm{t}} \chi_{m}^{0}$ \\
\hline
\end{tabular}

between $k$ th and $l$ th basis functions for $\mathrm{P}$ - and Q-type sources is the result of applying a Galerkin procedure to the basis expansions (6). Therefore, it can be also expressed as a modal sum

$$
R_{\mathrm{PQ}}(k, l)=\sum_{i} \tilde{G}_{\mathrm{PQ} i} C_{\mathrm{P}}(k, i) \bar{C}_{\mathrm{Q}}(l, i)
$$

where every modal function of (10) is now projected over each basis function from (6). Formally speaking, the coupling integral between the $k$ th basis function on the $i$ th mode is given by

$$
C_{\mathrm{P}}(k, i)=\int_{D_{\mathrm{P} k}} \mathbf{b}_{k}\left(u_{1}, u_{2}\right) \cdot \mathbf{p}_{i}\left(u_{1}, u_{2}\right) \mathrm{d} S .
$$

Thus far, only two assumptions have been made, namely, in (7) and (10), and the whole integration scheme in the integral equation-MoM problem has been reduced to the computation of coupling integrals. In the following section, we will demonstrate how these integrals can be systematically simplified. The reader should be aware of the fact that (12) does not refer to the coupling integrals solved in [9]-[11], since there the basis functions were eigensolutions of a predefined boundary problem. In that case, the coupling integrals can be solved using the procedure described in [19].

\section{GENERAL TRANSFORMATION OF COUPLING INTEGRALS}

The general expression for coupling integrals presented in (12) can take six different forms considering the combination of electric/magnetic sources with three different families of modes (TE, TM, and TEM), namely,

$$
\begin{aligned}
& C_{\mathrm{E}}^{\tau}=\int_{D_{\mathrm{E}}} \mathbf{b} \cdot \mathbf{e}^{\tau} \mathrm{d} S \\
& C_{\mathrm{H}}^{\tau}=\int_{D_{\mathrm{H}}} \mathbf{b} \cdot \mathbf{h}^{\tau} \mathrm{d} S
\end{aligned}
$$

with $\tau=\{$ TE, TM, TEM $\}$. We prefer to express these equations in terms of scalar potentials using the identities presented in Table I. A scalar potential $\chi$ (subindices are suppressed for simplicity) represents any eigensolution, with $\kappa$ being the associated eigenvalue, of the Helmholtz equation

$$
\left(\nabla_{\mathrm{t}}^{2}+\kappa^{2}\right) \chi=0
$$

with appropriate boundary conditions on the transverse boundary $\partial \Omega$ and where, as before, $\nabla_{t}^{2}=\nabla^{2}-\hat{z} \partial^{2} / \partial z^{2}$. If $\kappa=0$ (TEM case), the scalar potential, denoted in this case as $\chi^{0}$, is an eigensolution to the Laplace equation

$$
\nabla_{\mathrm{t}}^{2} \chi^{0}=0
$$


TABLE II

RELATIONS BETWEEN OVERLAPPING AND CONTOUR INTEGRALS

\begin{tabular}{|c|c|c|}
\hline Overlapping & Surface & Contour (b satisfies (7)) \\
\hline$\kappa C_{\mathrm{E}}^{\mathrm{TE}},-\kappa C_{\mathrm{H}}^{\mathrm{TM}},-C_{\mathrm{H}}^{\mathrm{TEM}}\left(\right.$ with $\left.\chi=\chi^{0}\right)$ & $\int_{D} \mathbf{b} \cdot\left(\hat{z} \times \nabla_{\mathrm{t}} \chi\right) \mathrm{d} S$ & $\oint_{\partial D} \chi b_{\tau} \mathrm{d} l$ \\
\hline$-\kappa C_{\mathrm{E}}^{\mathrm{TM}},-\kappa C_{\mathrm{H}}^{\mathrm{TE}}$ & $\int_{D} \mathbf{b} \cdot \nabla_{\mathrm{t}} \chi \mathrm{d} S$ & $\oint_{\partial D} \chi b_{\nu} \mathrm{d} l+\frac{d}{\kappa^{2}} \oint_{\partial D} \frac{\partial \chi}{\partial \nu} \mathrm{d} l, \quad \kappa \neq 0$ \\
$-C_{\mathrm{E}}^{\mathrm{TEM}}$ & $\oint_{\partial D} a \frac{\partial \chi^{0}}{\partial \nu} \mathrm{d} l$, & $\kappa=0$ \\
\hline \hline
\end{tabular}

Consequently, any integral in (13) can be written in terms of $\chi$ (analogously for $\left.\chi^{0}\right)$

$$
\begin{aligned}
& \int_{D} \mathbf{b} \cdot\left(\hat{z} \times \nabla_{\mathrm{t}} \chi\right) \mathrm{d} S=\int_{D}(\mathbf{b} \times \hat{z}) \cdot \nabla_{\mathrm{t}} \chi \mathrm{d} S \\
& \int_{D} \mathbf{b} \cdot \nabla_{\mathrm{t}} \chi \mathrm{d} S .
\end{aligned}
$$

These new expressions can now be further transformed into contour integrals using Green's first identity and the properties of the basis functions assumed in (7). Some details of the mathematical manipulations follow.

Green's first identity states that

$$
\int_{D} \mathbf{A} \cdot \nabla_{\mathrm{t}} B \mathrm{~d} S=\oint_{\partial D} B \hat{\nu} \cdot \mathbf{A} \mathrm{d} l-\int_{D} B \nabla_{\mathrm{t}} \cdot \mathbf{A} \mathrm{d} S .
$$

where $\left\{\mathbf{A}, \nabla_{\mathrm{t}} B\right\}$ represent two generic vector functions and $\hat{\nu}, \hat{\tau}$ are normal and tangential unit vectors defined over the integration contour $\partial D$.

Let us first demonstrate how (16a) is reduced to a contour integral. Substituting

$$
\mathbf{A}=\mathbf{b} \times \hat{z} \quad B=\chi
$$

into (17) results in an identity for (16). Using the relations $\hat{\nu}$. $(\mathbf{b} \times \hat{z})=\mathbf{b} \cdot \hat{\tau}$ and $\nabla_{\mathrm{t}} \cdot(\mathbf{b} \times \hat{z})=\left(\nabla_{\mathrm{t}} \times \mathbf{b}\right) \cdot \hat{z}$, we can rewrite (16a) as

$$
\int_{D} \mathbf{b} \cdot\left(\hat{z} \times \nabla_{\mathrm{t}} \chi\right) \mathrm{d} S=\oint_{\partial D} \chi \mathbf{b} \cdot \hat{\tau} \mathrm{d} l-\int_{D} \chi \nabla_{\mathrm{t}} \times \mathbf{b} \cdot \hat{z} \mathrm{~d} S .
$$

According to property (7a), the last term in the equation above vanishes, resulting in the desired transformation between surface and contour integrals. The demonstration is analogous for (16a) applied to the scalar potential $\chi^{0}$.

The manipulations needed on the integral (16b) differ depending on whether it involves $\chi$ or $\chi^{0}$. In the first case, (17) is used with

$$
\mathbf{A}=\mathbf{b} \quad B=\chi
$$

and ( $7 b)$ is applied to reduce the identity to

$$
\int_{D} \mathbf{b} \cdot \nabla_{\mathrm{t}} \chi \mathrm{d} S=\oint_{\partial D} \chi \mathbf{b} \cdot \hat{\nu} \mathrm{d} l-d \int_{D} \chi \mathrm{d} S .
$$

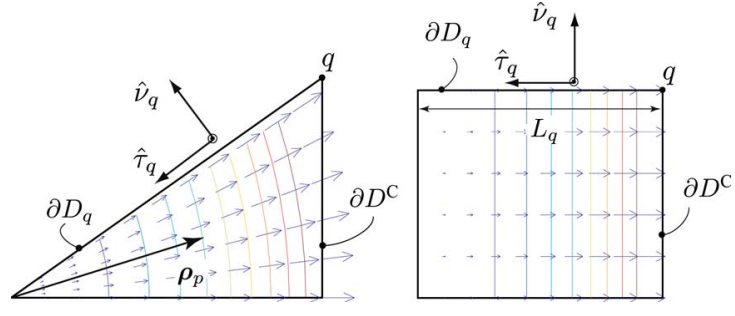

Fig. 2. Half-basis function $\mathbf{g}$ defined on a triangular and rectangular subdomain $D^{ \pm}$. The arrows represent the vector function $\mathbf{g}_{p}$, the lines the contour plot of the auxiliary function $f_{p}$, and the $q$ th nodes and sides are enumerated counterclockwise.

The remaining surface integral can be transformed using (14) as

$$
\chi=-\frac{\nabla_{\mathrm{t}}^{2} \chi}{\kappa^{2}}=-\frac{\nabla_{\mathrm{t}} \cdot\left(\nabla_{\mathrm{t}} \chi\right)}{\kappa^{2}}
$$

provided that $\kappa \neq 0$, and applying the Gauss theorem, i.e.,

$$
-\kappa^{2} \int_{D} \chi \mathrm{d} S=\int_{D} \nabla_{\mathrm{t}} \cdot\left(\nabla_{\mathrm{t}} \chi\right) \mathrm{d} S=\oint_{\partial D} \nabla_{\mathrm{t}} \chi \cdot \hat{\nu} \mathrm{d} l .
$$

Obviously, (18) cannot be applied to $\chi^{0}$ because it requires $\kappa \neq$ 0 . Instead, as the rotational in (7) vanishes, we can define an auxiliary function $a$ such that

$$
\begin{aligned}
\mathbf{b} & =\nabla_{\mathrm{t}} a \\
\nabla_{\mathrm{t}}^{2} a & =d .
\end{aligned}
$$

Then, using (17) with

$$
\mathbf{A}=\nabla_{\mathrm{t}} \chi^{0} \quad B=a
$$

we can rewrite (16b), for $\chi^{0}$, as

$$
\int_{D} \mathbf{b} \cdot \nabla_{\mathrm{t}} \chi^{0} \mathrm{~d} S=\oint_{\partial D} a \nabla_{\mathrm{t}} \chi^{0} \cdot \hat{\nu} \mathrm{d} l-\int_{D} a \nabla_{\mathrm{t}}^{2} \chi^{0} \mathrm{~d} S .
$$

Finally, since $\chi^{0}$ is a solution of (15), the surface integral on the right hand side of the previous equation vanishes.

The validity of these expressions extends to any kind of transverse boundary conditions that can be defined in terms of modal functions and to a large variety of basis functions having the properties in (7), among which we could mention the rooftop [20], the Rao-Wilton-Glisson [21] or the generalized PoissonNeumann polygonal basis functions [22]. A summary of the results in this section are given in Table II, with the following notation: $\partial \chi / \partial s \equiv \nabla_{\mathrm{t}} \chi \cdot \hat{s}$ and $\mathbf{b} \cdot \hat{s} \equiv b_{s}$ for $s=\nu, \tau$. 
TABLE III

VARIATION OF g-COMPONENTS ON A TRIANGUlAR (SECOND ROW) AND RECTANGULAR (THIRD Row) CONTOUR

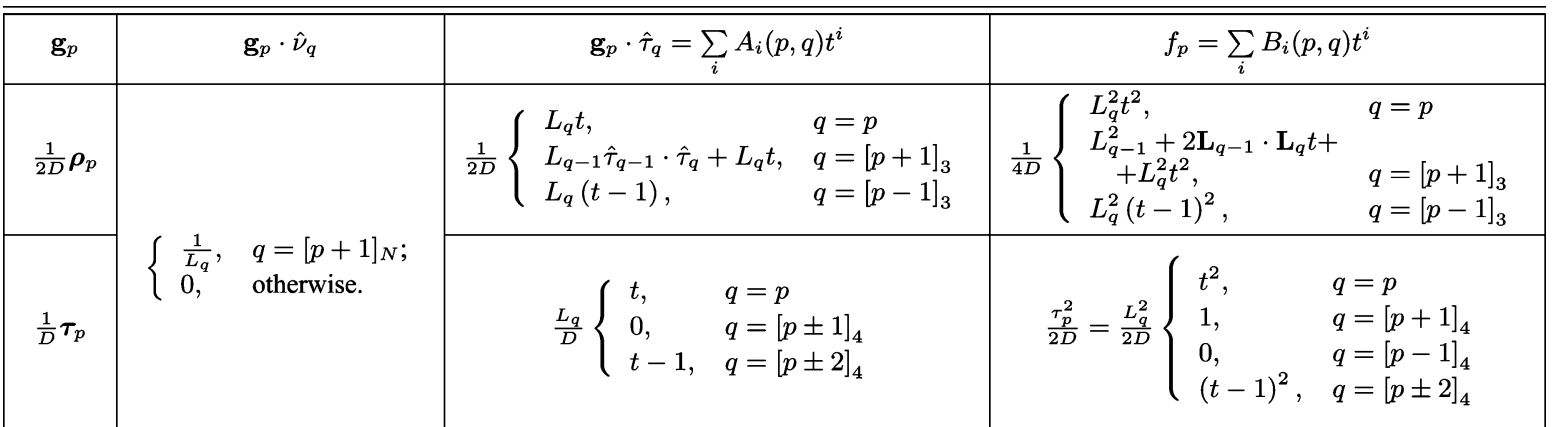

$p, q=0,1, \ldots N(=3,4)$ are 0 -based indices and $D$ is the subdomain's area

\section{COUPLing InTEgRals With Linear SUBSECTIONAL BASIS FUNCTIONS}

Linear subsectional basis functions, like rooftops [20] and Rao-Wilton-Glisson functions [21], constitute a common choice for the expansion of surface currents formulated in (6). Each planar surface $\mathcal{D}_{\mathrm{Q}}$ in the structure, as depicted in Fig. 1(b), is divided into triangular and/or rectangular subdomains that are used as support for these basis functions. Subsectional basis functions can be expressed as the sum of two "half-functions" with adjacent supports $D^{ \pm}$connected through a common side, denoted as $\partial D^{\mathrm{C}}$. In mathematical terms, the basis function $\mathbf{b}$ and its auxiliary function $a$, are decomposed into

$$
\mathbf{b}=\mathrm{g}^{+}-\mathrm{g}^{-} \quad a=f^{+}-f^{-}
$$

where $\mathbf{g}^{ \pm}, f^{ \pm} \in D^{ \pm}$and $D=D^{+} \cup D^{-}$.

In Fig. $2, \mathbf{g}^{ \pm}$and $f^{ \pm}$are represented on triangular and rectangular subdomains $D^{ \pm}$. The vector function $\mathbf{g}_{p}$ models a current flow diverging from the $p$ th node on the triangular subdomain, or in the direction of the $p$ th side if the subdomain is rectangular. The current varies linearly and is tangential to each side of $D^{ \pm}$ except along $\partial D^{\mathrm{C}}$, where it has a constant normal component $\mathrm{g} \cdot \hat{\tau}$ that guarantees the continuity of the current between adjacent subdomains in accordance with Kirchhoff's law.

The variation of these functions along the subdomain contour, regardless of its shape, can be expressed in a general form. Let us define a parametric function that maps a normalized variable $t$ into the $q$ th side of a subdomain as

$$
\begin{aligned}
\boldsymbol{\sigma}_{q}:[0,1] & \rightarrow D_{q} \subset \mathbb{R}^{2} \\
t & \rightsquigarrow \mathbf{r}(t)=\mathbf{P}_{q}+L_{q} t \hat{\tau}_{q}
\end{aligned}
$$

where $\mathbf{P}_{q}, L_{q} \hat{\tau}_{q}$ are vectors defined in Fig. 1. Therefore, the variation of any $p$ th "half-function" along any $q$ th side can be expressed as

$$
\begin{aligned}
& \mathbf{g}_{p}\left(\boldsymbol{\sigma}_{q}\right)=\left[A_{1}(p, q) t+A_{0}(p, q)\right] \hat{\tau}_{q}+\frac{\delta_{[p+1]_{N}, q}}{L_{q}} \hat{\nu}_{q} \\
& f_{p}\left(\boldsymbol{\sigma}_{q}\right)=B_{2}(p, q) t^{2}+B_{1}(p, q) t+B_{0}(p, q)
\end{aligned}
$$

where $A_{i}, B_{i} \in \mathbb{R}$ are constant values for each combination of $(p, q), N$ is the number of sides and $[x]_{y} \equiv \bmod (x, y)=x-n y$ where $n=\lfloor x / y\rfloor$ if $y \neq 0$ and $\delta_{[p+1]_{N}, q}$ designates a Kronecker delta that equals one when $q$ coincides with the common side.
The values of $A_{i}, B_{i}$ for triangular and rectangular contours can be found in Table III.

We now turn to the evaluation of coupling integrals using these types of basis functions. The contour form of coupling integrals presented in Table II is now calculated as the sum of line integrals along each side of the complete domain, i.e.,

$$
\oint_{\partial D} \cdots=\sum_{q=1}^{N}\left(\int_{\partial D_{q}} \cdots \mathrm{d} l\right)=\sum_{q=1}^{N} L_{q}\left(\int_{0}^{1} \cdots \mathrm{d} t\right)
$$

where each integral applies over a half basis function, as can be readily deduced from (20). Four types of line integrals can arise in (23) for a $q$ th side. Using (22), these can be written as

$$
\begin{aligned}
\int_{\partial D_{q}} \chi \mathbf{g}_{p} \cdot \hat{\nu}_{q} \mathrm{~d} l & =I_{q}(0) \delta_{[p+1]_{N}, q} \\
\int_{\partial D_{q}} \chi \mathbf{g}_{p} \cdot \hat{\tau}_{q} \mathrm{~d} l & =L_{q} \sum_{n=0}^{1} A_{n}(p, q) I_{q}(n) \\
\int_{\partial D_{q}} \frac{\partial \chi}{\partial \nu_{q}} \mathrm{~d} l & =L_{q} I_{q}^{\prime}(0) \\
\int_{\partial D_{q}} f_{p} \frac{\partial \chi^{0}}{\partial \nu_{q}} \mathrm{~d} l & =L_{q} \sum_{n=0}^{2} B_{n}(p, q) I_{q}^{\prime}(n)
\end{aligned}
$$

where

$$
\begin{aligned}
& I_{q}(n) \equiv \int_{0}^{1} t^{n} \chi\left(\boldsymbol{\sigma}_{q}(t)\right) \mathrm{d} t \\
& I_{q}^{\prime}(n) \equiv \int_{0}^{1} t^{n} \nabla_{\mathrm{t}} \chi\left(\boldsymbol{\sigma}_{q}(t)\right) \cdot \hat{\nu}_{q} \mathrm{~d} t
\end{aligned}
$$

for $n=0,1,2$. Note that the transition between the coupling integrals over any of the basis functions considered here is seamless and it is reduced to the proper choice of the constants $\left\{A_{i}, B_{i}\right\}$ in the formulation. In addition, the generality of the transverse boundary condition is still preserved in (23).

\section{SOME LATERAL BOUNDARY CONDITIONS}

The solution of the coupling integrals presented so far is subject to the election of appropriate boundary conditions, or equiv- 
alently, to the substitution of pertinent scalar potential functions in (25). In this section, we will obtain the solution to these integrals, and therefore to any coupling integrals using subsectional basis functions, in three practical boundary conditions, namely boundaries with rectangular and circular perfect electric conductor and a 2-D periodic boundary condition. In the following developments, the scale factor $\xi_{i}=\left[\int_{\Omega} \chi_{i} \chi_{i}^{*} \mathrm{~d} S\right]^{-1 / 2}$ is used as normalization of each $i$ th mode.

\section{A. Rectangular Perfect Electric Conductor}

Substituting the expressions for the $i$ th rectangular mode [16] into (25) and after some simple manipulations we obtain

$$
\begin{gathered}
I_{i, q}(n)=\frac{\xi_{i}}{2}\left[\int_{0}^{1} t^{n} \cos \Delta_{q} \mathrm{~d} t \pm \int_{0}^{1} t^{n} \cos \Sigma_{q} \mathrm{~d} t\right] \\
I_{i, q}^{\prime}(n)=-\frac{\xi_{i}}{2}\left[\left(\dot{\boldsymbol{\kappa}} \cdot \hat{\nu}_{q}\right) \int_{0}^{1} t^{n} \sin \Delta_{q} \mathrm{~d} t\right. \\
\left. \pm\left(\boldsymbol{\kappa} \cdot \hat{\nu}_{q}\right) \int_{0}^{1} t^{n} \sin \Sigma_{q} \mathrm{~d} t\right]
\end{gathered}
$$

where $n=0,1$ and $\Sigma_{q}, \Delta_{q}$ are

$$
\begin{aligned}
\Sigma & =k_{m} x+k_{n} y=\kappa \cdot \mathbf{r} \\
\Delta & =k_{m} x-k_{n} y=\dot{\boldsymbol{\kappa}} \cdot \mathbf{r}
\end{aligned}
$$

evaluated on the $q$ th side according to (21) (i.e., with $\mathbf{r}=\mathbf{P}_{q}+$ $\left.\mathbf{L}_{q} t\right)$.

\section{B. Circular Perfect Electric Conductor}

The integrals (25) are transformed using the circular mode expresions in [16] in conjunction with the cylindrical to cartesian transformation presented in [23] that approximates

$$
\begin{aligned}
\chi_{i} \propto J_{m}\left(\kappa_{i} r\right) \exp (\mathrm{j} m \varphi) \approx \frac{\mathrm{j}^{m}}{N} \sum_{l=0}^{N-1} \exp \left(\mathrm{j} l \frac{2 m \pi}{N}\right) \\
\cdot \exp \left(-\mathrm{j} \kappa_{i} \mathbf{T}_{l} \cdot \mathbf{r}\right)
\end{aligned}
$$

where

$$
\mathbf{T}_{l} \cdot \mathbf{r}=x \cos \frac{2 l \pi}{N}+y \sin \frac{2 l \pi}{N}
$$

and $N-1>\kappa_{i} r+N_{0}$ with $N_{0}$ being a small integer. The final expressions for (25) are

$$
\begin{aligned}
& \left.\begin{array}{c}
I_{i, q}(n) \\
I_{i, q}^{\prime}(n)
\end{array}\right\}=\frac{\mathrm{j}^{m} \xi_{i}}{N} \sum_{l=0}^{N-1} \exp \left[\mathrm{j}\left(l \frac{2 m \pi}{N}-\kappa_{i} \mathbf{T}_{l} \cdot \mathbf{P}_{q}\right)\right] \\
& \times\left\{\begin{array}{l}
\int_{0}^{1} t^{n} \exp \left(-\mathrm{j} \kappa_{i} \mathbf{T}_{l} \cdot \mathbf{L}_{q} t\right) \mathrm{d} t \\
-\mathrm{j} \kappa_{i} \mathbf{T}_{l} \cdot \hat{\nu}_{q} \int_{0}^{1} t^{n} \exp \left(-\mathrm{j} \kappa_{i} \mathbf{T}_{l} \cdot \mathbf{L}_{q} t\right) \mathrm{d} t
\end{array}\right.
\end{aligned}
$$

for $n=0,1$.

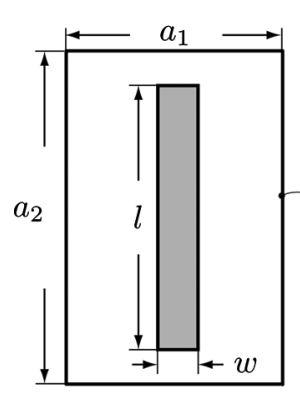

(a)

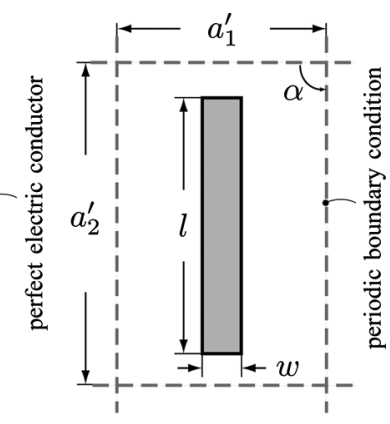

(b)
Fig. 3. Planar strip conductor of $w=2.38 \mathrm{~mm}$ and $l=13.30 \mathrm{~mm}$ used as: (a) an iris in a rectangular waveguide of $a_{1}=7.60 \mathrm{~mm}, a_{2}=15.20 \mathrm{~mm}$ and (b) a unit cell in a 2-D periodic lattice with $a_{1}^{\prime}=7.60 \mathrm{~mm}, a_{2}^{\prime}=15.20 \mathrm{~mm}$ and $\alpha=90^{\circ}$.

\section{General Periodic Lattice}

Let us consider a periodic structure with translation vectors in the direct and reciprocal lattices [24] given by

$$
\boldsymbol{\rho}_{m n}=m \mathbf{a}_{1}+n \mathbf{a}_{2}
$$

and

$$
\begin{aligned}
\mathbf{k}_{m n} & =m \mathbf{k}_{1}+n \mathbf{k}_{2} \\
& =\frac{2 m \pi}{a_{1}} \hat{x}+\left(\frac{2 n \pi}{a_{2} \sin \alpha}-\frac{2 m \pi}{a_{1} \tan \alpha}\right) \hat{y}
\end{aligned}
$$

respectively. The structure is illuminated with an arbitrary polarized plane wave impinging with angles $(\theta, \phi)$ and

$$
\mathbf{k}=k \sin \theta \cos \phi \hat{x}+k \sin \theta \sin \phi \hat{y}
$$

where $k=\omega \sqrt{\mu \varepsilon}$ is the propagation constant. Equation (25) for the $i$ th Floquet mode [17] can be expressed as

$$
\begin{aligned}
& I_{i, q}(n)=\xi_{i} \exp \left(\mathrm{j} \boldsymbol{\kappa}_{i} \cdot \mathbf{P}_{q}\right) \int_{0}^{1} t^{n} \exp \left(\mathrm{j} \boldsymbol{\kappa}_{i} \cdot \mathbf{L}_{q} t\right) \mathrm{d} t \\
& I_{i, q}^{\prime}(n)=\mathrm{j} \boldsymbol{\kappa}_{i} \cdot \hat{\nu}_{q} I_{i, q}(n)
\end{aligned}
$$

where $\boldsymbol{\kappa}_{i} \equiv \boldsymbol{\kappa}_{m n}=\mathbf{k}_{m n}-\mathbf{k}$ and $n=0,1,2$.

The remaining integrals in(26), (28), and(29) have trivial analytic solution.

\section{NUMERICAL EXAMPLES}

In the first two examples, represented in Fig. 3, we consider a planar strip conductor surrounded by two different transverse boundary conditions, namely a rectangular perfect electric conductor and periodic boundary condition. The first structure, in Fig. 3(a), is excited with the mode $\mathrm{TE}_{01}$ of the rectangular waveguide. The return and insertion losses at $z=0^{ \pm}$are computed using different methods and the results are compared in Fig. 4. Two simulations have been performed with the methods presented using different mesh schemes in each case.

The same structure has also been simulated with a different integral equation-based method described in [25] and an approach based on the mode matching/generalized transverse resonance technique [26]. The agreement between the two integral equation approaches is excellent while the differences arising 


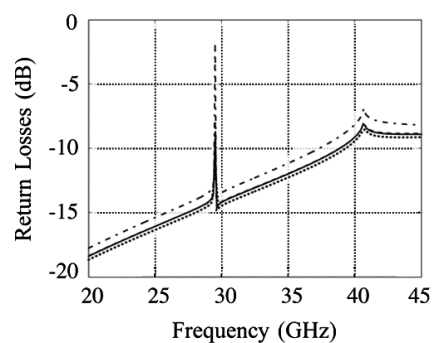

(a)

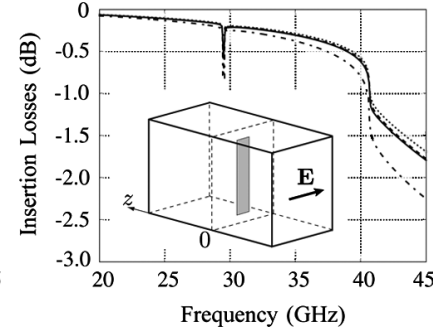

(b)
Fig. 4. Scattering of the $\mathrm{TE}_{01}$ mode from a strip inside a rectangular waveguide in Fig. 3(a). The presented method using only rooftops (solid line) or Rao-Wilton-Glisson basis functions (dashed line), integral equation method of [25] (dotted line) and mode matching/generalized transverse resonance technique of [26] (dashed-dotted line).

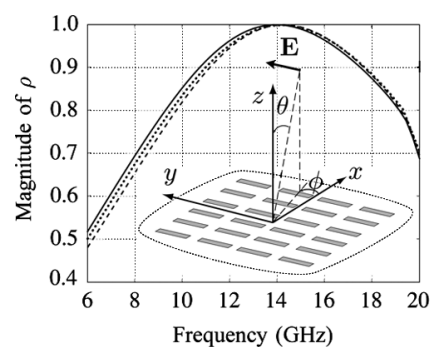

(a)

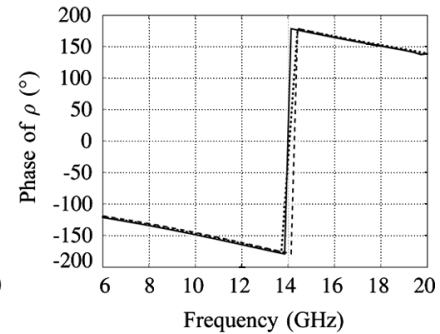

(b)
Fig. 5. Reflection coefficient $\rho$ at $z=0^{+}$for a $T E_{00}$ Floquet mode impinging the periodic lattice Fig. 3(b) with angles $\theta=1^{\circ}, \phi=1^{\circ}$. This method using only rooftops (solid line) or Rao-Wilton-Glisson basis functions (dashed line) and simulations from [27] (dotted line).

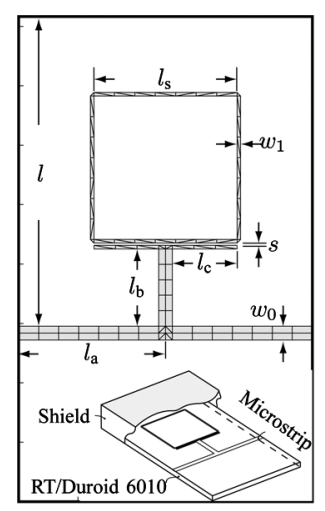

(a) Circuit layout.

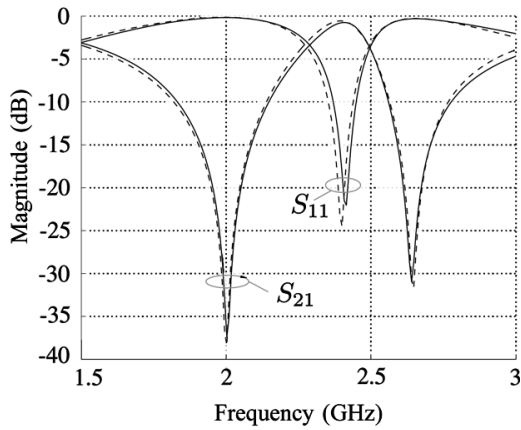

(b) Magnitude of scattering parameters.
Fig. 6. Return and insertion losses simulated with the presented method (solid line) and MoM based software (dashed line). The dimensions of the circuit are $l_{\mathrm{s}}=12.07 \mathrm{~mm}, s=0.20 \mathrm{~mm}, l_{\mathrm{a}}=12.38 \mathrm{~mm}, l_{\mathrm{b}}=6.50 \mathrm{~mm}, l_{\mathrm{c}}=$ $5.46 \mathrm{~mm}, w_{0}=1.16 \mathrm{~mm}, w_{1}=0.3 \mathrm{~mm}, l=26.06 \mathrm{~mm}$. The metallic shield is $24.75 \mathrm{~mm} \times 40.83 \mathrm{~mm} \times 5.27 \mathrm{~mm}$ and the dielectric is RT/Duroid 6010 with a thickness of $1.27 \mathrm{~mm}$.

as compared to the mode matching/generalized transverse resonance method are associated to the zero-thickness nature of the structure. The same planar strip is used as a unit cell in a planar periodic structure in Fig. 3(b). The structure is illuminated with a $\mathrm{TE}_{00}$ mode slightly tilted with respect to the normal direction. The scattering is presented in Fig. 5 and compared with the results provided in [27] showing an excellent agreement in phase and differences of at most $7 \%$ in magnitude. Finally, this method has also been applied in the simulation of a more realistic structure involving a two-port microstrip resonator enclosed in a metallic rectangular shield, as represented in Fig. 6(a). The response shown in Fig. 6(b) is simulated with this method and Ansoft Designer [28], a MoM-based commercial software. This result demonstrates how this method can also be applied to multilayer structures and hybrid meshes, providing accurate results.

\section{CONCLUSIONS}

We have presented a new integral equation-MoM formulation, based on modal expansion, for the EM modeling of laterally bounded planar structures in multilayered media. Essential to this method is the strategy used for the resolution of coupling integrals combining divergence conforming basis functions, with locally zero-curl and constant-charge, and modal functions derived from the transverse boundary problem. The coupling integrals have been first reduced to contour integrals in a general fashion. Then, these were specialized to linear subsectional basis functions over triangular or rectangular domains with a seamless transition between the two. An example of application showed solutions for three different boundary conditions. This technique avoids having a specific formulation for each type of problem. Instead, it offers a unified and versatile approach that, on the one hand eliminates redundancy in the formulation and on the other hand reduces each particular problem to the proper choice of constant coefficients or the evaluation of a few basic line integrals. In particular, seemingly different problems like discontinuities in circular waveguides and 2-D periodic structures embedded in multilayered media, are brought under the same frame. Once properly implemented, the technique does not introduce any computational overhead when compared with other standard integral equation-MoM approaches, while widely broadening the scope of solvable problems. Finally, the method has been validated with several numerical examples and the results compared against alternative approaches. This work also opens the door for obvious extensions, like the treatment of more general boundaries with planar or curved contours. This generalization should be easily integrated into the present framework and work towards this goal is under progress.

\section{REFERENCES}

[1] J. L. Volakis, A. Chatterjee, and L. C. Kempel, Finite Element Method for Electromagnetics. Piscataway, NJ: IEEE Press, 1998.

[2] A. Taflove and S. C. Hagness, "Advances in Computational Electrodynamics," in The Finite-Difference Time-domain Method, A. Taflove, Ed., 3rd ed. Norwood, MA: Artech House, 2005.

[3] C. Christopoulos, "The transmission-line modeling method in electromagnetics," in Synthesis Lectures on Computational Electromagnetics, C. A. Balanis, Ed. New York: Morgan Claypool, 2006.

[4] J. R. Mosig, "Integral-equation technique," in Numerical Techniques for Microwave and Millimeter-Wave Passive Structures, T. Itoh, Ed. New York: Wiley, 1989, ch. 3, pp. 133-213.

[5] W. C. Chew, Waves and Fields in Inhomogenous Media, ser. Electromagn. Wave Theory. Piscataway, NJ: IEEE Press, 1990.

[6] B. M. Kolundzija and A. R. Djordjević, Electromagnetic Modeling of Composite Metallic and Dielectric Structures. Boston, MA: Artech House, 2002.

[7] C. Kim, S. Yu, R. Harrington, J. Ra, and S. Lee, "Computation of waveguide modes for waveguides of arbitrary cross-section," Proc. Inst. Elect. Eng.-Microw., Antennas, Propag., vol. 137, no. 2, pt. H, pp. 145-149, Apr. 1990. 
[8] A. F. Peterson, S. L. Ray, and R. Mittra, Computational Methods for Electromagnetics. Piscataway, NJ: IEEE Press, 1998.

[9] A. A. Melcón, J. R. Mosig, and M. Guglielmi, "Efficient CAD of boxed microwave circuits based on arbitrary rectangular elements," IEEE Trans. Microw. Theory Tech., vol. 47, no. 7, pp. 1045-1058, Jul. 1999.

[10] M. Bozzi and L. Perregrini, "Analysis of multilayered printed frequency selective surfaces by the MoM/BI-RME method," IEEE Trans. Antennas Propag., vol. 51, no. 10, pp. 2830-2836, Oct. 2003.

[11] M. Bozzi, L. Perregrini, A. A. Melcón, M. Guglielmi, and G. Conciauro, "MoM/BI-RME analysis of boxed MMICs with arbitrarily shaped metallizations," IEEE Trans. Microw. Theory Tech., vol. 49, no. 12, pp. 2227-2234, Dec. 2001.

[12] R. E. Collin, Field Theory of Guided Waves, 2nd ed ed. Piscataway, NJ: IEEE Press, 1990.

[13] R. F. Harrington, Field Computation by Moments Method. New York: Macmillan, 1968.

[14] V. H. Rumsey, "Reaction concept in electromagnetic theory," Phys. Rev., vol. 94, pp. 1483-1491, Jun. 1954.

[15] L. B. Felsen and N. Marcuvitz, Radiation and Scattering of Waves. Englewood Cliffs, NJ: Prentice-Hall, 1973.

[16] N. Marcuvitz, Waveguide Handbook, ser. Radiat. Lab. New York: McGraw-Hill, 1941.

[17] C.-C. Chen, "Transmission through a conducting screen perforated periodically with apertures," IEEE Trans. Microw. Theory Tech., vol. MTT-18, no. 9, pp. 627-632, Sep. 1970.

[18] K. A. Michalski and J. R. Mosig, "Multilayered media Green's functions in integral equation formulations," IEEE Trans. Antennas Propag., vol. 45, no. 3, pp. 508-519, Mar. 1997.

[19] G. Figlia and G. Gentili, "On the line-integral formulation of modematching technique," IEEE Trans. Microw. Theory Tech., vol. 50, no. 2, pp. 578-580, Feb. 2002.

[20] A. Glisson and D. Wilton, "Simple and efficient numerical methods for problems of electromagnetic radiation and scattering from surfaces," IEEE Trans. Antennas Propag., vol. AP-28, no. 5, pp. 593-603, Sep. 1980.

[21] S. Rao, D. Wilton, and A. Glisson, "Electromagnetic scattering by surfaces of arbitrary shape," IEEE Trans. Antennas Propag., vol. 30, no. 5, pp. 409-418, May 1982.

[22] L. Knockaert, J. Sercu, and D. de Zutter, "Generalized Poisson-Neumann polygonal basis functions for the electromagnetic simulation of complex planar structures," IEEE Trans. Microw. Theory Tech., vol. 52, no. 3, pp. 954-961, Mar. 2004.

[23] R. H. MacPhie and K.-L. Wu, "Scattering at the junction of a rectangular waveguide and a larger circular waveguide," IEEE Trans. Microw. Theory Tech., vol. 43, no. 9, pp. 2041-2045, Sep. 1995.

[24] L. Brillouin, Wave Propagation in Periodic Structures. New York: Dover, 1953, ch. VI, pp. 94-130.

[25] I. Stevanović, P. Crespo-Valero, and J. R. Mosig, "An integral-equation technique for solving thick irises in rectangular waveguides," IEEE Trans. Microw. Theory Tech., vol. 54, no. 1, pp. 189-197, Jan. 2006.

[26] J. A. Ruiz-Cruz, M. A. Sabbagh, K. A. Zaki, J. Rebollar, and Y. Zhang, "Canonical ridge waveguide filters in LTCC or metallic resonators," IEEE Trans. Microw. Theory Tech., vol. 53, no. Jan., pp. 174-182, Jan. 2005.

[27] C. H. Chan and R. Mittra, "On the analysis of frequency-selective surfaces using subdomain basis functions," IEEE Trans. Antennas Propag., vol. 38, no. 1, pp. 40-50, Jan. 1990.

[28] Designer Ansoft Corporation, Pittsburgh, PA, 2005. [Online]. Available: http://www.ansoft.com/products/hf/ansoft_designer/

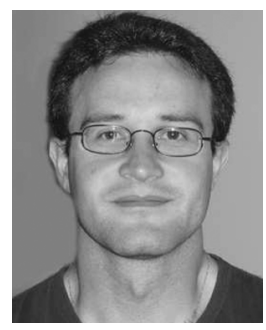

Pedro Crespo-Valero was born in Antas (Almería), Spain, in 1976. He received the Ingeniero de Telecomunicación M.Sc. degree from the Universidad Politécnica de Madrid (UPM), Madrid, Spain, in 2001, and the Ph.D. degree from the École Polytechnique Fédérale de Lausanne (EPFL), Lausanne, Switzerland, in 2007.

He was a member of Colegio Mayor Diego de Covarrubias, Universidad Complutense de Madrid (UCM), Madrid, Spain. In October 2007, he joined the Research and Development Software Group,
Schmid \& Partner Engineering AG (SPEAG), Zurich, Switzerland, where he is currently involved in the development of SEMCAD X, a professional thermal and EM simulation tool.

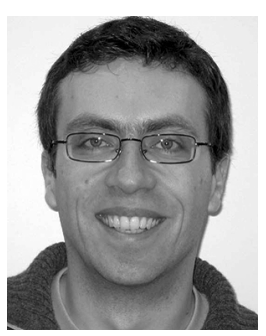

Ivica Stevanović (S'03-M'05) was born in Kruševac, Serbia, in 1976. He received the Dipl.Ing. degree in electrical engineering from the University of Belgrade, Belgrade, Serbia, in 2000, and the $\mathrm{Ph} . \mathrm{D}$. degree in electrical engineering from the Ecole Polytechnique Fédérale de Lausanne (EPFL), Lausanne, Switzerland, in 2005.

In 2000, he was a SURF Research Fellow with the Laser Interferometer Gravitational Wave Observatory, California Institute of Technology, Pasadena. From 2000 to 2006, he was with the Laboratory of Electromagnetics and Acoustics, EPFL, where he was a Research and Teaching Assistant (2000-2005) and a Post-Doctoral Research Fellow (2005-2006). Since 2006, he has been Senior Research and Development Engineer with Freescale Semiconductor, Geneva, Switzerland.

Dr. Stevanović was the recipient of an NSF-REU-SURF Research Fellowship presented by the California Institute of Technology in 2000.

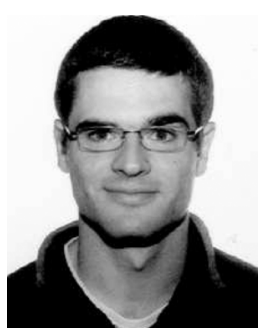

Daniel Llorens del Río (M’07) received the Telecommunications Engineer degree from the University of Málaga, Málaga, Spain, in 2000, and the $\mathrm{Ph} . \mathrm{D}$. degree from the École Polytechnique Fédérale de Lausanne (EPFL), Switzerland, in 2005.

Since 2006, he has been with JAST Antenna Systems, Lausanne, Switzerland. His main interests are antenna design and numerical modeling.

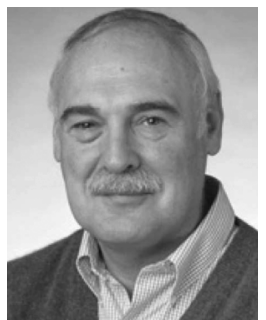

Juan R. Mosig (S'76-M'87-SM'94-F'99) was born in Cádiz, Spain. He received the Electrical Engineer degree from the Universidad Politécnica de Madrid, Madrid, Spain, in 1973, and the Ph.D. degree from the École Polytechnique Fédérale de Lausanne (EPFL), Lausanne, Switzerland, in 1983.

In 1976, he joined the Laboratory of Electromagnetics and Acoustics, EPFL. Since 1991, he has been a Professor with EPFL, and since 2000, he has been the Head of the Laboratory of Electromagnetics and Acoustics (LEMA), EPFL. In 1984, he was a Visiting Research Associate with the Rochester Institute of Technology, Rochester, NY, and Syracuse University, Syracuse, NY. He has also held scientific appointments with the University of Rennes, Rennes, France, the University of Nice, Nice, France, the Technical University of Denmark, Lyngby, Denmark, and the University of Colorado at Boulder. He is currently the Chairman of the EPFL Space Center and is responsible for many Swiss research projects for the European Space Agency (ESA). He has authored five book chapters on microstrip antennas and circuits and over 100 reviewed papers. His research interests include EM theory, numerical methods, and planar antennas.

Dr. Mosig has been a member of the Swiss Federal Commission for Space Applications. He is currently a member of the Board of the Applied Computational Electromagnetics Society (ACES), the chairman of the European COST Project on Antennas ASSIST (2007-2011), and a founding member and acting chair of the European Association and the European Conference on Antennas and Propagation (EurAAP and EuCAP). 